\title{
Propagation of thermal waves with lateral heat transfer
}

\author{
R.K. Sahoo \\ Mechanical Engineering Department, Regional Engineering College, Rourkela 769008 \\ (Orissa), India
}

Presently with National Institute of Technology, Rourkela, India rksahoo@nitrkl.ac.in

The wave nature of heat propagation in a one-dimensional semi-infinite medium with lateral convective heat transfer is investigated by solving the hyperbolic heat conduction equation in the longitudinal direction. The situation involves a large relaxation time which is relevant at low temperatures or for materials with a nonhomogeneous inner structure, and the heat conduction in the lateral direction is assumed to be parabolic due to the number of lateral heat wave reflections compared to those in the longitudinal direction. The results for a unit heat flux condition at the boundary are compared with those obtained from parabolic heat conduction. This reveals that the classical heat diffusion theory predicted by parabolic heat conduction significantly underestimates the magnitude of the temperature and heat flux in thermal wave propagation. The results also reveal that when the dimensionless lateral heat transfer, known as the wave shape factor, is equal to unity, the thermal wavefront which travels through the medium at a finite speed decays exponentially along its path of travel while retaining the shape of the input wave. This theoretical prediction can be implemented experimentally for estimation of thermal relaxation times.

Keywords: thermodynamics; heat transfer; thermal waves

\begin{tabular}{|c|c|c|c|}
\hline \multicolumn{2}{|c|}{ Nomenclature } & $\bar{T}_{\mathrm{D}}$ & Transformed dimensionless temperature \\
\hline$A$ & Cross-sectional area of medium $\left(\mathrm{m}^{2}\right)$ & $u$ & Intermediate variable for time \\
\hline$C$ & Specific heat of medium $\left(\mathrm{J} \mathrm{kg}^{-1} \mathrm{~K}^{-1}\right)$ & $U$ & Unit step function \\
\hline$g(t)$ & Function in time & $v$ & $\begin{array}{l}\text { Speed of propagation of thermal wave } \\
{[=\sqrt{(\alpha / \tau)}]\left(\mathrm{m} \mathrm{s}^{-1}\right)}\end{array}$ \\
\hline$G(s)$ & & $x$ & Spatial co-ordinate $(\mathrm{m})$ \\
\hline $\begin{array}{l}h \\
K\end{array}$ & $\begin{array}{l}\text { Heat transfer coefficient }\left(\mathrm{W} \mathrm{m}^{-2} \mathrm{~K}^{-1}\right) \\
\text { Thermal conductivity }\left(\mathrm{W} \mathrm{m}^{-1} \mathrm{~K}^{-1}\right)\end{array}$ & $x_{\mathrm{D}}$ & Dimensionless spatial variable $[=x / \sqrt{(\alpha \tau)}]$ \\
\hline$P$ & & $z$ & Intermediate variable $\left[=\left(u^{2}-x_{D}^{2}\right)^{1 / 2}\right]$ \\
\hline$q$ & Heat flux $\left(\mathrm{W} \mathrm{m}^{-2}\right)$ & $Z$ & Intermediate constant $\left[=\left(t_{\mathrm{D}}^{2}-x_{\mathrm{D}}^{2}\right)^{1 / 2}\right]$ \\
\hline$q_{\mathrm{D}}$ & Dimensionless heat flux $\left(=q / q_{0}\right)$ & & \\
\hline$\tilde{q}_{\mathrm{D}}$ & Transformed dimensionless heat flux & \multirow{2}{*}{\multicolumn{2}{|c|}{ Greek letters }} \\
\hline$q_{0}$ & Input heat flux $\left(\mathrm{W} \mathrm{m}^{-2}\right)$ & & \\
\hline$t$ & $\begin{array}{l}\text { Laplace transtorm varıable } \\
\text { Time co-ordinate (s) }\end{array}$ & $\alpha$ & Thermal diffusivity $[=K / \rho C]\left(\mathrm{m}^{2} \mathrm{~s}^{-1}\right)$ \\
\hline$t_{\mathrm{D}}$ & Dimensionless time variable $(=t / \tau)$ & $\beta$ & Intermediate variable $[=\sqrt{(s+\phi)(s+1)}]$ \\
\hline$T$ & Temperature (K) & $\rho$ & Density of medium $\left(\mathrm{kg} \mathrm{m}^{-3}\right)$ \\
\hline$T_{0}$ & Initial temperature of medium $(\mathrm{K})$ & $\tau$ & Thermal relaxation time $(\mathrm{s})$ \\
\hline$T_{\mathrm{D}}$ & $\begin{array}{l}\text { Dimensionless temperature } \\
{\left[=\left(T-T_{0}\right) \sqrt{(\rho C K / \tau)} / q_{0}\right]}\end{array}$ & $\phi$ & $\begin{array}{l}\text { Wave shape factor or dimensionless heat } \\
\text { dissipation factor }[=h P \tau / A \rho C]\end{array}$ \\
\hline
\end{tabular}

When considering transient heat flow, for short times, extreme thermal gradients or temperatures approaching absolute zero (liquid helium), the classical heat diffusion theory predicted by Fourier breaks down. In the classical theory of heat conduction, the heat flux is postulated to be directly proportional to the temperature gradient

$q=-K \operatorname{grad} T$

When this flux law is combined with the following 
energy conservation equation (without internal or external heat interaction)

$\rho C \frac{\partial T}{\partial t}=-\operatorname{div} q$

the heat conduction becomes parabolic in nature. The resultant parabolic heat conduction equation, given by

$$
\frac{\partial T}{\partial t}=\alpha \nabla^{2} T
$$

is a diffusion phenomenon which is predicted by Fourier. It shows that a thermal disturbance at any distant point in a medium will be instantaneously felt at every point in that medium. Despite this physically unrealistic notion of instantaneous heat diffusion with infinite propagation speed, the heat flow predictions given by Equation (3) are quite reliable for most situations encountered in practice.

Strictly speaking, the Fourier law of heat conduction should be applied to low rate, steady state transfer processes, and physically speaking there must be a time scale for which the validity of this law is violated. In situations dealing with transient heat flow of extremely short duration, for that short time of higher input temperature or heat flux pulses at the boundary, the classical heat diffusion theory becomes invalid. Also, at very low temperatures the rapid rate of heat conduction due to excited phonons forbids the use of the Fourier law. In these situations, heat has been observed to travel as a wave with a finite propagation speed. Using superfluid helium at a temperature near absolute zero, Peshkov ${ }^{1}$ in 1944 experimentally detected the existence of these thermal waves. He referred to this phenomenon as 'second sound' because of the similarity in wave velocity between these thermal waves and ordinary acoustic waves. The phenomenon of 'second sound' persists in solid as well as liquid helium due to the presence of excited phonon gas.

To incorporate the finite propagation speed while retaining the basic nature of the Fourier law, Morse and Feshbach ${ }^{2}$ in 1953 and later Vernotte Ve $^{3,4}$ in 1958 and Cattaneo $^{5}$ in 1958 formulated a modified heat flux law originally suggested by Maxwell ${ }^{6}$ in 1867 , in the form

$$
q+\tau \quad \frac{\partial q}{\partial t}=-K \nabla T
$$

When the flux law of the form given by Equation (4) is used in conjunction with the conservation law [Equation (2)] a hyperbolic heat conduction equation results, as opposed to the classical parabolic equation based on the flux law [Equation (1)]. It is reported by Chester ${ }^{7}$ that Equation (4) is actually a truncated form of an extensive relation originally derived by Maxwell ${ }^{6}$ from kinetic theory considerations. By applying a different approach based on irreversible thermodynamics, Luikov ${ }^{8}$ also arrived at the same conclusion.

In Equation (4), $\tau$ represents the relaxation time or build-up period for the initiation of heat flow after a temperature gradient has been imposed at the boundary of the medium. It states that heat flow does not start instantaneously, but rather grows gradually with a relaxation time $\tau$ after the application of the temperature gradient. Conversely, there is a time lag for the disappearance of the heat flow after the removal of the temperature gradient. In fact, the relaxation time $\tau$ is associated with the communication 'time' between phonons (phonon-phonon collisions) necessary for commencement of heat flow and is a measure of the thermal inertia of the medium. A typical value of the relaxation time $\tau$ for metals at ambient temperature has been reported ${ }^{9}$ to be of the order of $10^{-11} \mathrm{~s}$, while recent work by Kaminski ${ }^{10}$ on materials with a nonhomogeneous inner structure revealed values of $\tau$ of the order of fractions of a minute. When the relaxation time $\tau$ is relatively large, as is the case for temperatures near absolute zero, thermal disturbances appear to propagate as waves at finite speeds, as observed experimentally by Peshkov ${ }^{1}$ and Bertman and Sandiford ${ }^{11}$. At a critical point the limit situation $\tau \rightarrow 0$ leads to instantaneous diffusion at an infinite propagation velocity, which coincides with classical diffusion theory.

Advances in research on heat transfer at a finite propagation velocity have resulted in increased interest in the solution of hyperbolic heat conduction. Analytical and numerical solutions concerning certain models are given in the literature for some specific cases.

The wavefront resulting from a step change in temperature at the boundary of a medium is demonstrated by Mikic $^{12}$ and Baumeister and Hamill ${ }^{13,14}$. The solution to the problem due to a step change in heat flux at the boundary is given by Maurer and Thompson ${ }^{15}$, while its numerical solution using the method of characteristics is obtained by Wiggert ${ }^{16}$. When the region of a finite medium is considered, the thermal disturbance in the medium becomes a superimposed effect of forward and backward travelling waves. The backward travelling waves are the reflections off the boundaries. These problems, with different boundary conditions, are solved by Taitel ${ }^{17}$ and Carey and Tsai $^{18}$.

With respect to the analysis of propagation of thermal disturbances in a medium containing distributed volumetric energy sources, it is important to compare the experimental results obtained by Bertman and Sandiford ${ }^{11}$. In this respect, the analysis for a semiinfinite medium is given by Vick and Ozisik ${ }^{19}$, while that for a finite medium is given by Ozisik and Vick ${ }^{20}$, Frankel et al. ${ }^{21}$ and Gembarovic and Majernik ${ }^{9}$.

For situations in which analytical solutions are difficult to obtain, as in the case with radiation or with temperature-dependent thermal conductivity, hyperbolic heat conduction equations are solved by Glass and Ozisik, with McRae and Vick ${ }^{22,23}$ and with Vick ${ }^{24}$. An exhaustive literature review on the development of ideas about heat waves is presented by Joseph ${ }^{25}$.

For hyperbolic heat conduction dealing with small values of $\tau\left(\approx 10^{-11} \mathrm{~s}\right)$, the wave phenomenon is observed over a small region $\left(\approx 10^{-8} \mathrm{~m}\right)^{9}$, and beyond this region parabolic heat conduction prevails. So convective heat transfer from the lateral surface becomes unrealistic. But at higher values of $\tau$ the wave phenomenon is observed over an appreciable part of the medium. There is a gap in the literature on reported values of $\tau$ for solids at cryogenic temperatures. Apart from solid helium ${ }^{26}$, some alkali halide crystals $^{27}$ are promising in this respect. One of the most promising and widely used dielectric materials is diamond. It has been recently reported ${ }^{28}$ that diamond 
has a high relaxation time $\tau\left(\approx 10^{-3} \mathrm{~s}\right)$ at liquid nitrogen temprature $(77 \mathrm{~K})$ and exhibits hyperbolic heat conduction in a macroscopic sample, as observed over an appreciable part of the medium.

In a one-dimensional semi-infinite medium, as in the case of a one-dimensional long fin, the lateral surface is exposed to a convective environment. Thus, during heat flux wave propagation in the lateral direction, a certain amount of heat flux is dissipated in the medium itself, while some is transmitted through the convective boundary, and the rest is reflected back to the medium. As the lateral 'length' is very small compared to that in the longitudinal direction, there will be a number of reflections of the heat wave in the lateral direction before the wave travels an appreciable distance in the longitudinal direction. Since each reflection in the lateral direction dissipates a certain amount of heat flux in the medium itself, it may be assumed that heat conduction along in this direction resumes its parabolic nature. Also, due to the smaller lateral dimension, we can assume that the temperature is constant for a low temperature gradient. So the lateral heat transfer is governed by convection between that constant temperature in the medium and the ambient temperature.

There appears to be no work in the literature addressing such a phenomenon. At cryogenic or high temperatures this phenomenon becomes severe. Thus the objective of the present investigation is to develop solutions for the temperature and heat flux as predicted by hyperbolic heat conduction in a one-dimensional long fin subjected to parabolic lateral heat transfer with a step input of heat flux at the boundary.

\section{Hyperbolic heat conduction}

The present investigation concerns a one-dimensional semi-infinite medium with lateral heat transfer, where one-dimensional heat conduction and constant thermal properties prevail. The medium is initially in equilibrium at an ambient temperature $T_{0}$ and the analysis is focussed on the propagation of a thermal disturbance initiated by a step input of heat flux at the boundary. The system and its control volume are illustrated schematically in Figure 1.

\section{Governing equations}

The one-dimensional conservation equation for rectangular co-ordinates with lateral heat transfer, as shown in Figure I, may be written, by modifying Equation (2), as

$$
\frac{\partial q}{\partial x}+\rho C \frac{\partial T}{\partial t}+\frac{h P}{A}\left(T-T_{0}\right)=0
$$

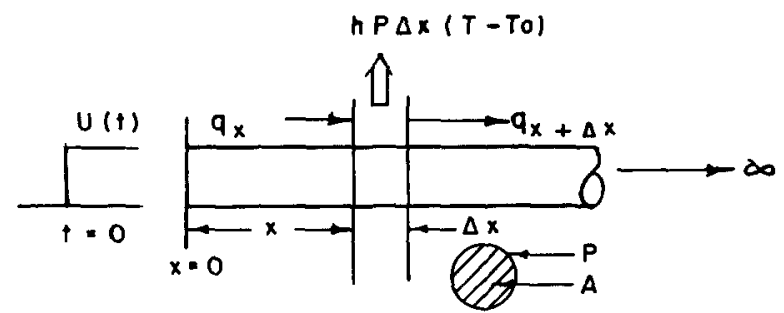

Figure 1 Schematic diagram of system (see text for details) where $h$ represents the heat transfer coefficient between the medium and its environment, and $P$ and $A$ are the perimeter and cross-sectional area, respectively. The modified heat flux equation given by Equation (4) may be written for a one-dimensional case as

$K \frac{\partial T}{\partial x}+\tau \frac{\partial q}{\partial x}+q=0$

Equations (5a) and (5b) are the coupled hyperbolic partial differential equations for analysis of the hyperbolic heat conduction problem. These equations are subjected to the initial conditions

$$
\begin{aligned}
& T(x, 0)=T_{0} \\
& q(x, 0)=0
\end{aligned}
$$

and the boundary conditions

$$
\begin{aligned}
& q(0, t)=q_{0} U(t) \\
& q(x, t) \rightarrow 0 ; x \rightarrow \infty
\end{aligned}
$$

Using the method of characteristics ${ }^{16}$, the velocity of propagation of the wavefront is given by

$\frac{\mathrm{d} x}{\mathrm{~d} t}=v=(\alpha / \tau)^{1 / 2}$

For convenience in the subsequent analysis, the following dimensionless quantities are defined:

dimensionless distance, $x_{\mathrm{D}}=x /(\alpha \tau)^{1 / 2}$

dimensionless time, $t_{\mathrm{D}}=t / \tau$

dimensionless temperature, $T_{\mathrm{D}}=\left(T-T_{0}\right)$

$$
\times(\rho C K / \tau)^{1 / 2} / q_{0}
$$

dimensionless heat flux, $q_{\mathrm{D}}=q / q_{0}$

wave shape factor, $\phi=h P \tau / A \rho C$

With these dimensionless quantities, the set of Equations (5), (6) and (7) are reduced to Equations $(8),(9)$ and (10), respectively, as

$$
\begin{aligned}
& \frac{\partial q_{\mathrm{D}}}{\partial x_{\mathrm{D}}}+\frac{\partial T_{\mathrm{D}}}{\partial t_{\mathrm{D}}}+\phi T_{\mathrm{D}}=0 \\
& \frac{\partial T_{\mathrm{D}}}{\partial x_{\mathrm{D}}}+\frac{\partial q_{\mathrm{D}}}{\partial t_{\mathrm{D}}}+q_{\mathrm{D}}=0 \\
& T_{\mathrm{D}}\left(x_{\mathrm{D}}, 0\right)=0 \\
& q_{\mathrm{D}}\left(x_{\mathrm{D}}, 0\right)=0 \\
& q_{\mathrm{D}}\left(0, t_{\mathrm{D}}\right)=U\left(t_{\mathrm{D}}\right) \\
& q_{\mathrm{D}}\left(x_{\mathrm{D}}, t_{\mathrm{D}}\right) \rightarrow 0 ; x_{\mathrm{D}} \rightarrow \infty
\end{aligned}
$$

The solution to the above governing system of equations is developed in the following section.

\section{Analysis}

The system defined by Equations (8), (9) and (10) can be solved by the application of a Laplace transform with respect to temporal variables. The appropriate subsidiary equations are obtained from Equations (8a) and $(8 b)$ by the application of the initial conditions 
given by Equations (9a) and (9b). These equations are

$$
\begin{aligned}
& \frac{\mathrm{d} \tilde{q}_{\mathrm{D}}}{\mathrm{d} x_{\mathrm{D}}}=-\bar{T}_{\mathrm{D}}(s+\phi) \\
& \frac{\mathrm{d} \bar{T}_{\mathrm{D}}}{\mathrm{d} x_{\mathrm{D}}}=-\bar{q}_{\mathrm{D}}(s+1)
\end{aligned}
$$

with the boundary conditions

$\bar{q}_{\mathrm{D}}(0, s)=1 / s$

$\bar{q}_{\mathrm{D}}\left(x_{\mathrm{D}}, s\right) \rightarrow 0 ; x_{\mathrm{D}} \rightarrow \infty$

where

$\bar{q}_{\mathrm{D}}=\int_{0}^{\infty} q_{\mathrm{D}}\left(x_{\mathrm{D}}, t_{\mathrm{D}}\right) \exp \left(-s t_{\mathrm{D}}\right) \mathrm{d} t_{\mathrm{D}}$

and

$\bar{T}_{\mathrm{D}}=\int_{0}^{\infty} T_{D}\left(x_{\mathrm{D}}, t_{\mathrm{D}}\right) \exp \left(-s t_{\mathrm{D}}\right) \mathrm{d} t_{\mathrm{D}}$

By eliminating dimensionless temperature from the subsidiary Equations (11a) and (11b), the equation for dimensionless heat flux is obtained as

$\frac{\mathrm{d}^{2} \bar{q}_{\mathrm{D}}}{\mathrm{d} x_{\mathrm{D}}^{2}}=\bar{q}_{\mathrm{D}}(s+\phi)(s+1)$

The solution to Equation (13) for the boundary conditions given by Equations (12a) and (12b) is

$\tilde{q}_{\mathrm{D}}=(1 / s) \exp \left(-\beta x_{\mathrm{D}}\right)$

From Equations (11a) and (14a) the dimensionless temperature is derived as

$\bar{T}_{\mathrm{D}}=\frac{\beta}{s(s+\phi)} \exp \left(-\beta x_{\mathrm{D}}\right)$

where $\beta^{2}=(s+\phi)(s+1)$.

Using the table of Laplace transforms ${ }^{29}$, the inverse transform of Equations (14a) and (14b) is expressed as

$$
\begin{aligned}
q_{\mathrm{D}}= & U\left(t_{\mathrm{D}}-x_{\mathrm{D}}\right)\left\{\exp \left[-x_{\mathrm{D}}(\phi+1) / 2\right]+(\phi-1)\left(x_{\mathrm{D}} / 2\right)\right. \\
& \left.\times \int_{x_{\mathrm{D}}}^{t_{\mathrm{D}}}\left(\exp [-(\phi+1) u / 2] \frac{I_{1}(\phi-1) z / 2}{z}\right) \mathrm{d} u\right\}
\end{aligned}
$$

and

$$
\begin{aligned}
T_{\mathrm{D}}=U\left(t_{\mathrm{D}}-x_{\mathrm{D}}\right)\left\{\exp \left[-t_{\mathrm{D}}(\phi+1) / 2\right] I_{0}[(\phi-1) Z / 2]\right. \\
\left.+\int_{x_{\mathrm{D}}}^{t_{\mathrm{D}}}\left(\exp [-(\phi+1) u / 2] I_{0}[(\phi-1) z / 2]\right) \mathrm{d} u\right\}
\end{aligned}
$$

where $Z^{2}=t_{\mathrm{D}}^{2}-x_{\mathrm{D}}^{2}$ and $z^{2}=u^{2}-x_{\mathrm{D}}^{2} \cdot I_{0}$ and $I_{1}$ are modified Bessel functions of the first kind for zero order and first order, respectively.

It is of practical interest to evaluate the following two limiting cases for dimensionless temperature.
Case 1: $x_{D} \rightarrow 0$

The dimensionless surface temperature can be obtained either from Equation (14b) or from Equation (15b) by putting $x_{\mathrm{D}}=0$, as

$$
\begin{gathered}
T_{\mathrm{D}}\left(0, t_{\mathrm{D}}\right)=\exp \left[-t_{\mathrm{D}}(\phi+1) / 2\right] I_{0}\left[(\phi-1) t_{\mathrm{D}} / 2\right] \\
\quad+\int_{0}^{t_{\mathrm{D}}} \exp [-(\phi+1) u / 2] I_{0}[(\phi-1) u / 2] \mathrm{d} u
\end{gathered}
$$

\section{Case 2: $\mathrm{x}_{D} \rightarrow 0$ and $\mathrm{t}_{D} \rightarrow 0$}

The dimensionless initial surface temperature can be obtained from Equation (16) in the $t_{\mathrm{D}} \rightarrow 0$ limit as

$\lim _{t_{\mathrm{D}} \rightarrow 0} T_{\mathrm{D}}\left(0, t_{\mathrm{D}}\right)=1$

This relation can also be obtained directly from Equation (14b) at $x_{D}=0$ by using the Laplace transform theorem

$\operatorname{Lim}_{t \rightarrow 0} g(t)=\operatorname{Lim}_{s \rightarrow \infty} s G(s)$

where $G(s)$ is the Laplace transform of function $g(t)$.

The latter solution indicates that a solid subjected to a step change in the surface heat flux with lateral heat transfer also experiences an initial jump in surface temperature. The solution to the hyperbolic heat conduction equation without lateral heat transfer for a step input of heat flux can be derived from Equations (15a) and (15b) by substituting $\phi=0$. The resultant equations are

$$
\begin{aligned}
q_{\mathrm{D}}=U\left(t_{\mathrm{D}}-x_{\mathrm{D}}\right)\left\{\exp \left(-x_{\mathrm{D}} / 2\right)\right. \\
\left.\quad+\left(x_{\mathrm{D}} / 2\right) \int_{x_{\mathrm{D}}}^{t_{\mathrm{D}}}\left[\exp (-u / 2) \frac{I_{1}(z / 2)}{z}\right] \mathrm{d} u\right\}
\end{aligned}
$$

and

$$
\begin{aligned}
T_{\mathrm{D}}=U\left(t_{\mathrm{D}}-x_{\mathrm{D}}\right) & \left\{\exp \left(-t_{\mathrm{D}} / 2\right) I_{0}(Z / 2)\right. \\
& \left.+\int_{x_{\mathrm{D}}}^{t_{\mathrm{D}}}\left[\exp (-u / 2) I_{0}(z / 2)\right] \mathrm{d} u\right\}
\end{aligned}
$$

where $Z^{2}=t_{\mathrm{D}}^{2}-x_{\mathrm{D}}^{2}$ and $z^{2}=u^{2}-x_{\mathrm{D}}^{2} . \quad I_{0}$ and $I_{1}$ represent modified Bessel functions of the first kind for zero order and first order, respectively. These equations are the same as those derived by Maurer and Thompson ${ }^{15}$.

\section{Significance of wave shape factor $\phi$}

The dimensionless lateral heat transfer parameter plays an important role in the analysis of hyperbolic heat conduction. It is interesting to note that for $\phi$ equal to unity, Equations (15a) and (15b) reduce to

$$
\begin{aligned}
& q_{\mathrm{D}}=\exp \left(-x_{\mathrm{D}}\right) U\left(t_{\mathrm{D}}-x_{\mathrm{D}}\right) \\
& T_{\mathrm{D}}=\exp \left(-x_{\mathrm{D}}\right) U\left(t_{\mathrm{D}}-x_{\mathrm{D}}\right)
\end{aligned}
$$


This result indicates that for a unit step input of dimensionless heat flux, the temperature and heat flux itself propagate in the medium as square waves which decay by a factor $\exp \left(-x_{D}\right)$ over a distance $x_{D}$.

So the shape of the input wave is retained by the propagating waves in the medium. In general, for $\phi=1$, if the input of dimensionless heat flux is given by some general function $f\left(t_{\mathrm{D}}\right)$, then the response can be easily derived from Equations (11a) and (1b) as

$q_{\mathrm{D}}=\exp \left(-x_{\mathrm{D}}\right) f\left(t_{\mathrm{D}}-x_{\mathrm{D}}\right) U\left(t_{\mathrm{D}}-x_{\mathrm{D}}\right)$

and

$T_{\mathrm{D}}=\exp \left(-x_{\mathrm{D}}\right) f\left(t_{\mathrm{D}}-x_{\mathrm{D}}\right) U\left(t_{\mathrm{D}}-x_{\mathrm{D}}\right)$

In these general solutions also the heat flux and temperature retain their shape but the magnitudes decay exponentially. To be more general, if instead of heat flux, a step input of temperature is applied at the boundary, an analogous situation prevails. Due to this peculiar nature of $\phi$, by which the input wave shape is reproduced, it is referred to as the 'wave shape factor' by the present author. An experimental method for determination of the relaxation time $\tau$ based on this theory is described in a later section.

According to heat transfer terminology, $\phi$ may be defined as the ratio of energy transfer laterally over the relaxation time to the internal energy stored by the medium. Since relaxation time $\tau$ has a wide range of values, it may be greater than, equal to or less than $A \rho C / h P$, depending on the material properties and heat transfer characteristics. Thus $h P \tau / A \rho C$ yields the corresponding dimensionless values of $\phi$.

\section{Parabolic heat conduction}

Though the solution of parabolic heat conduction with lateral heat transfer is not the essential aspect of the present investigation, its importance is recognized for comparison with hyperbolic heat conduction. The solutions for parabolic heat conduction based on the present dimensionless parameters and boundary conditions are not directly available in the literature. Hence it will be useful to review the solution procedure on the basis of the current analysis. The solution procedure for parabolic heat conduction is given in the Appendix.

\section{Results and discussion}

Numerical computations for the hyperbolic and parabolic heat conduction equations are performed to examine the behaviour of the temperature and heat flux distributions. For comparative purposes, both cases are plotted on the same figure. The equations involved for hyperbolic heat conduction are (15a) and (15b) along with the limiting cases described by Equations (16), (17), (18a) and (18b); whereas in parabolic heat conduction, equations (A5a) and (A5b) along with its limiting cases described by equations (A6a), (A6b) and (A6c) are utilized.

Figure 2 shows the variation of surface temperature with time. The temperature for hyperbolic heat conduction either increases, decreases or remains constant, depending on whether the wave shape factor $\phi$ is less

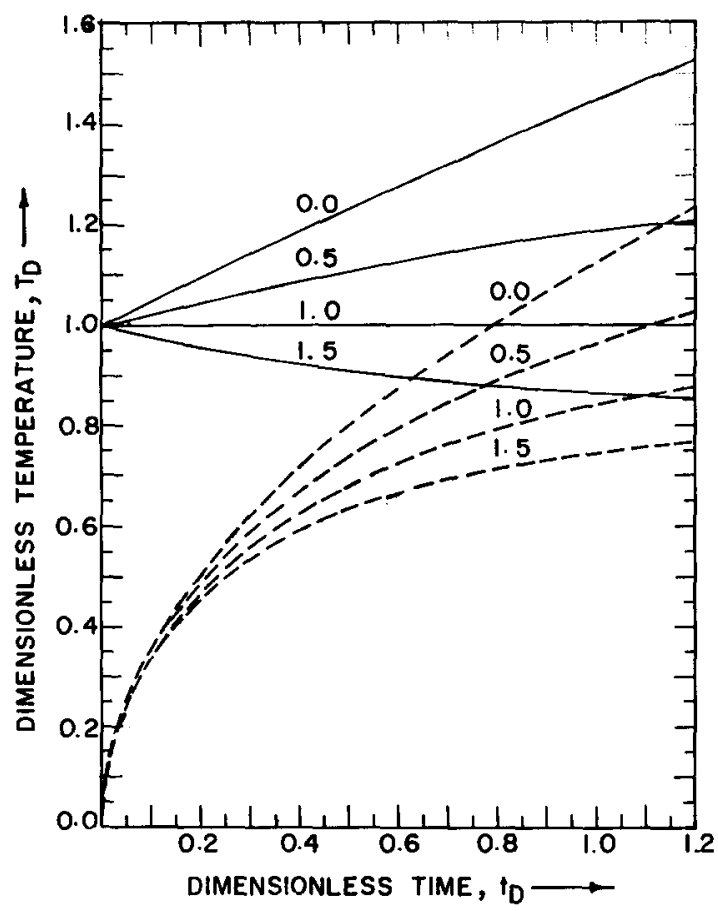

Figure 2 Dimensionless surface temperature profiles $\left(x_{D}=0\right)$ versus dimensionless time for ---, parabolic and hyperbolic heat conduction equations. Numbers given are wave shape factor $\phi$

than, greater than or equal to unity. This results from the imbalance between input heat flux and lateral heat dissipation. In particular, for a wave shape factor $\phi$ equal to unity, the lateral heat loss is just sufficiently balanced to maintain a constant temperature profile. But the net heat flux decreases or increases depending on $\phi$ being greater than or less than untiy. This net heat flux in the medium results in a decrease or increase in the temperature profile.

The uniqueness of the hyperbolic mode of heat conduction is to produce an initial surface temperature jump at all values of $\phi$, as evident from Equation (16) and Figure 2. In the other set of curves in the same figure, parabolic heat conduction builds up from the initial zero reference temperature with time, but with increasing values of $\phi$ the temperature profiles become more flattened due to a greater amount of lateral heat dissipation.

Figure 3 depicts temperature versus position at selected values of time (i.e. $t_{\mathrm{D}}=0.2,0.5$ and 1.0 ). To show the effects of lateral heat dissipation, this graph is repeated in Figures $3 a, b, c$ and $d$ for a wave shape factor $\phi$ of $0.0,0.5,1.0$ and 1.5 , respectively. The striking feature of these graphs is that for hyperbolic heat conduction energy input in terms of heat flux gives rise to a thermal wavefront which travels in the medium at a finite velocity and decays exponentially, while dissipating its energy along its path. In Figure $3 a$ this dissipation of energy is through the medium itself due to the adiabatic lateral surface $(\phi=0.0)$. But for other values of $\phi$, additional energy dissipation takes place across the lateral surface. So, with increasing values of $\phi$, the energy dissipation increases and hence, the amplitude of the thermal wave decreases at any particular point in time.

The most interesting feature of these plots is that for 

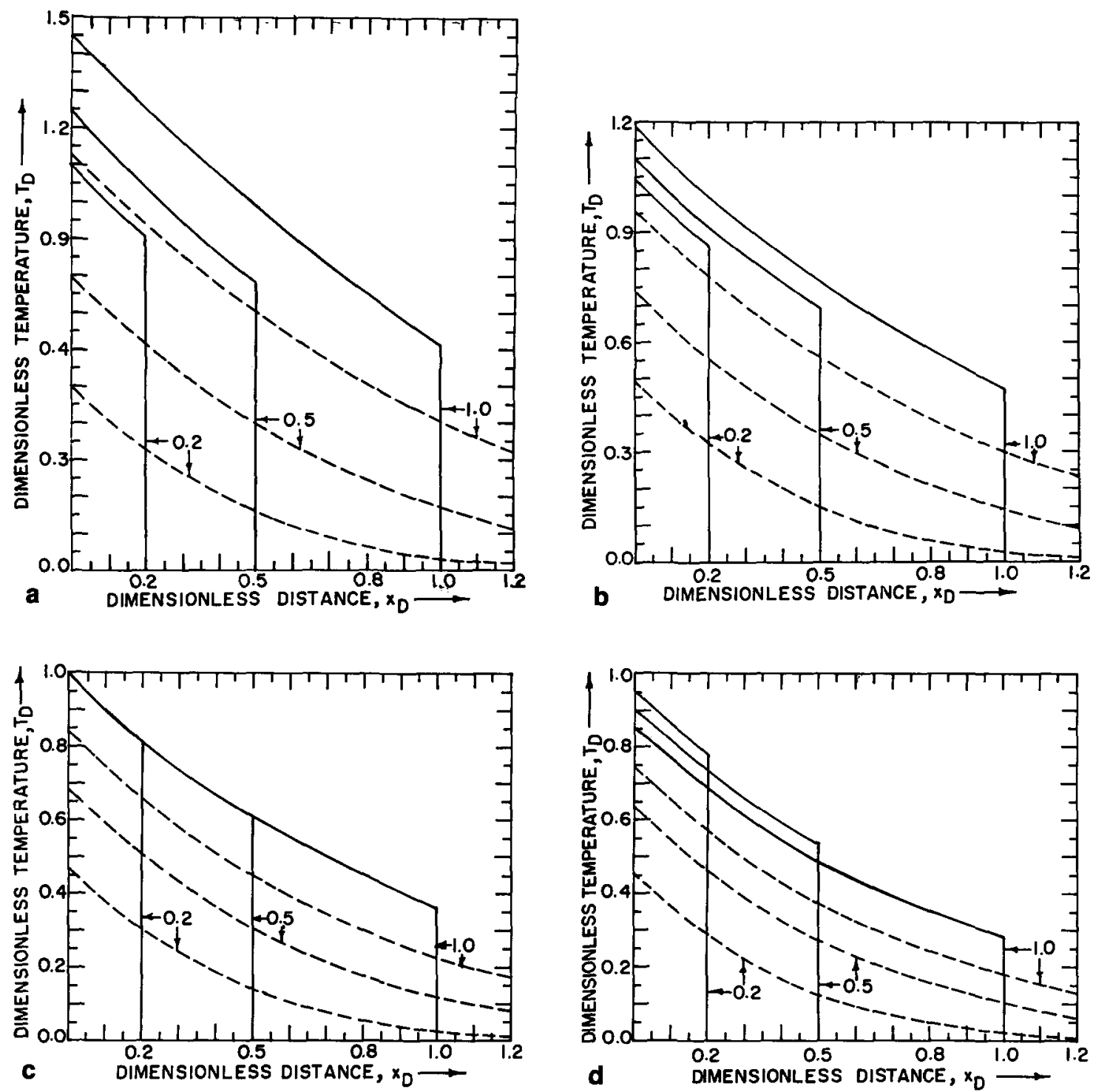

Figure 3 Dimensionless temperature profiles versus dimensionless distance with various wave shape factors: (a) $\phi=0.0$; (b) $\phi=0.5$; (c) $\phi=1.0$; (d) $\phi=1.5$, for - - , parabolic and - hyperbolic heat conduction equations. Numbers given are dimensionless time

a wave shape factor $\phi$ less than unity the temperature curves shift down with increasing time, as shown in Figures $3 a$ and $b$, whereas for a wave shape factor $\phi$ greater than unity this trend is reversed, as shown in Figure $3 d$. But in the case of a wave shape factor $\phi$ equal to unity, all the temperature profiles merge to form a single curve, though the wavefronts are at different positions, as shown in Figure 3c. This behaviour of the temperature profile is due to the surface temperature, as described earlier in Figure 2, at the origin of the thermal wavefront. For example, the behaviour of the temperature profile in Figure $3 d$ is due to the decreasing nature of the surface temperature in Figure 2 for $\phi>1.0$. Irrespective of the temperature variation with $\phi$, these plots exhibit propagation of the thermal wavefront in the medium at a finite speed and the medium is undisturbed beyond this wavefront. In contrast to this phenomenon, parabolic heat conduction shows a 'down-shifting' of the temperature profiles with increasing values of time and wave shape factor $\phi$, due to a proportional increase in lateral heat dissipation.
Figure 4 shows the temperature response at positions $x_{\mathrm{D}}=0.1,0.4$ and 0.8 with increasing values of wave shape factor $\phi$ (i.e. $\phi=0.0,0.5,1.0$ and 1.5 ) on the respective figures (Figures $4 a-d$ ). Hyperbolic heat conduction indicates that the temperature wave is not instantaneously felt at any point in the medium, but that there is a time lag before the wavefront travels to that point. This time lag for the onset of the thermal wave is independent of $\phi$. For a wave shape factor $\phi$ either greater than, equal to or less than unity, the temperature profile changes its shape accordingly. The temperature builds up for $\phi<1$, remains stationary for $\phi=1$ and decays for $\phi>1$, as shown in Figures $3 a-d$. This observation is supported by the fact that at $\phi=1$, the lateral heat dissipation is just sufficient to maintain a steady state temperature, whereas at $\phi$ greater than or less than unity, the net heat in the medium is deficient or in surplus, respectively. These figures also indicate that hyperbolic heat conduction approaches the parabolic state at higher values of time, and this phenomenon accelerates with increasing values of $\phi$. Hence, it may be inferred that at higher values of wave 

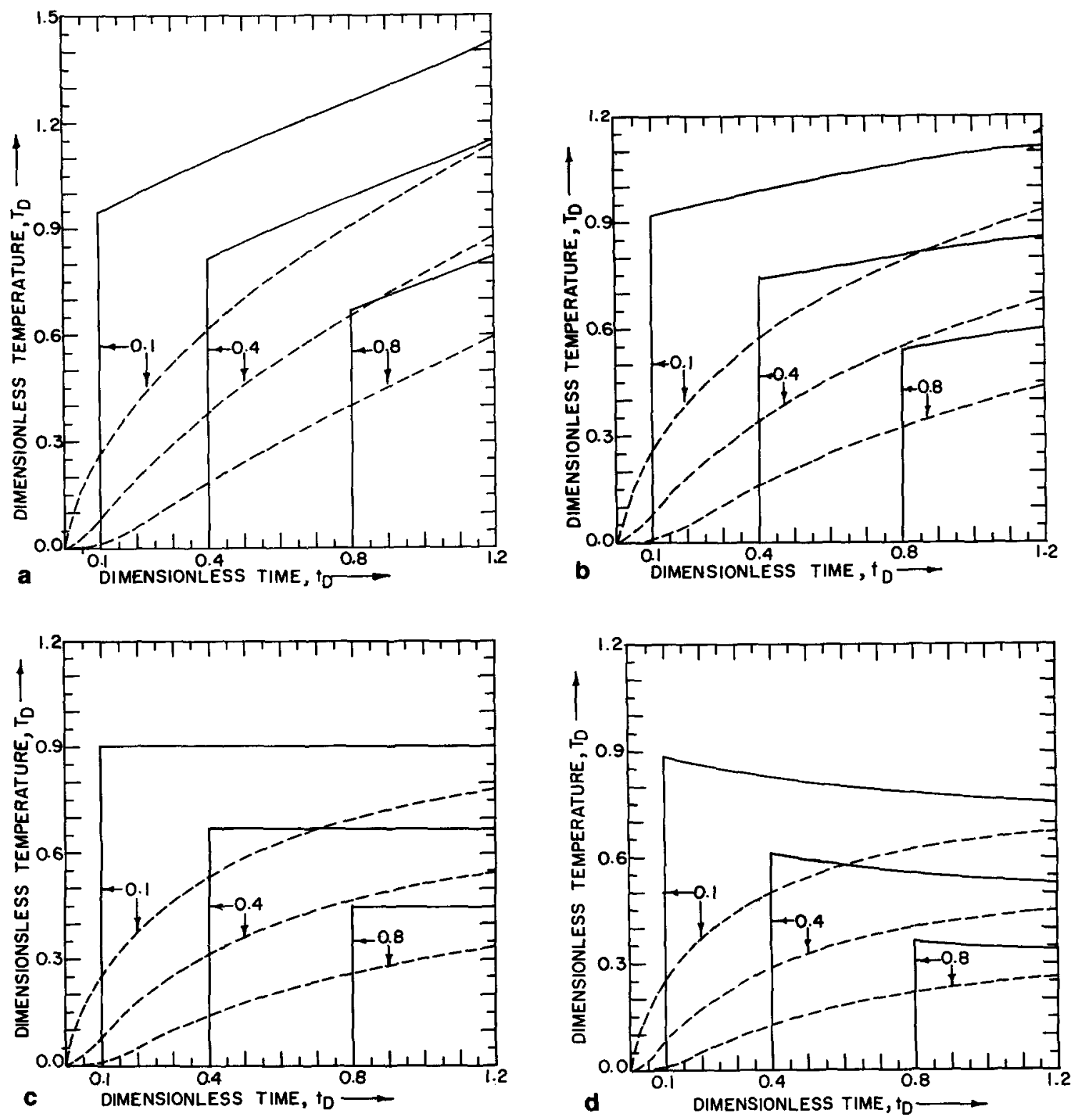

Figure 4 Dimensionless temperature profiles versus dimensionless time with various wave shape factors: (a) $\phi=0.0$; (b) $\phi=0.5$; (c) $\phi=1.0$; (d) $\phi=1.5$, for --- , parabolic and - hyperbolic heat conduction equations. Numbers given are dimensionless distance

shape factor $\phi$, the wave nature of thermal propagation is retained by an appropriate time delay but at later times the wave nature of thermal propagation behaves as a diffusion-like phenomenon.

Figures 5 and 6 are drawn in a similar way to Figures 3 and 4 to show the flux distribution predicted by the hyperbolic and parabolic heat conduction equations at given values of $\phi$. In Figure 5 the heat flux is plotted versus position at various times and in Figure 6 these two independent parameters are interchanged. The temperature solution described in Figures 3 and 4 is the result of the application of heat flux as a boundary condition. Unlike this solution, Figures 4 and 5 illustrate the propagation of the imposed boundary heat flux itself.

As shown in Figures $5 a$ and $b$, hyperbolic heat conduction portrays flux wave propagation through the medium at a finite speed. The wave decays exponentially along its path of travel by dissipating energy along the medium in addition to lateral heat dissipation. The presence of lateral heat dissipation is due to a non-zero value of $\phi$. With a wave shape factor equal to unity, Figure $5 b$ exhibits a single flux profile where the wave travels to different positions depending on the duration of wave propagation. This observation is similar to that described for the temperature solution in Figure $3 c$. For comparison, the parabolic heat conduction curves are drawn in the same figures to show the diffusive nature of heat conduction.

As shown in Figures $6 a$ and $b$, hyperbolic heat conduction portrays the time delay for the initiation of the heat flux wave at a position in the medium. The most interesting part of this analysis is the shape of the flux wave depicted in Figure $6 b$. This figure indicates that hyperbolic heat conduction reproduces the shape of the input heat flux at any position for $\phi=1$. Though the magnitude of the heat flux decays with position, its shape is retained. When heat flux propaga- 

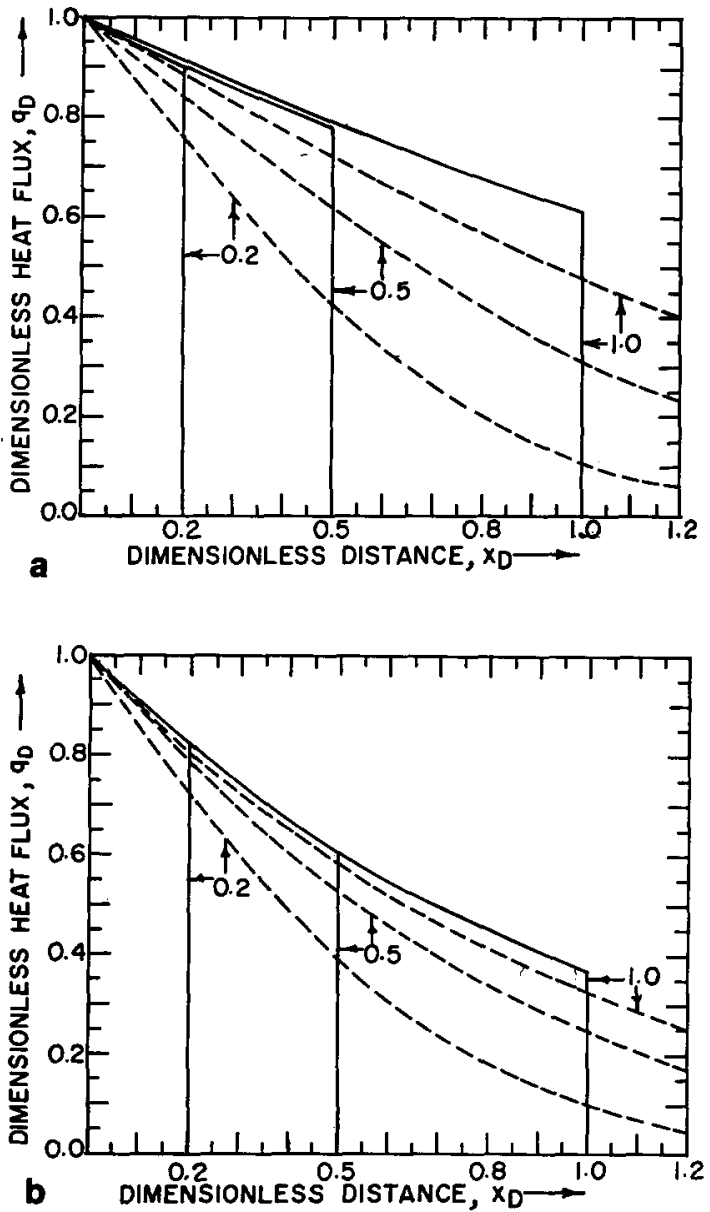

Figure 5 Dimensionless heat flux versus dimensionless distance with various wave shape factors: (a) $\phi=0.0$; (b) $\phi=$ 1.0, for - - , parabolic and - hyperbolic heat conduction equations. Numbers given are dimensionless time

tion is compared for both cases of heat conduction, these figures show that hyperbolic heat conduction approaches the parabolic state earlier with an increasing wave shape factor $\phi$, i.e. with increasing lateral convection. These observations are similar to the temperature solution described in Figure 4.

Recently, several questions have arisen in connection with the experiments involving the determination of relaxation time. The present analysis utilizes a novel technique to estimate the relaxation time from heat transfer measurements. The heat transfer coefficient used in this analysis is based on an average value over the spatial and temporal co-ordinates for a similar situation of unsteady state parabolic heat conduction. In general, the Biot or Nusselt number gives an estimation of the heat transfer coefficient which can be varied by the influence of the Reynolds and Prandtl number in the functional correlations. Following from Figure $6 b$, the lateral heat transfer coefficient can be adjusted to reproduce the shape of the input heat flux on an oscilloscope at the specified position in a long conducting medium. By measuring the decrement in flux amplitude, the relaxation time can be calculated from Equation (19a). However the same technique can be applied if, instead of heat flux, the temperature gradient is applied at the boundary.

It is important to note that the presence of lateral
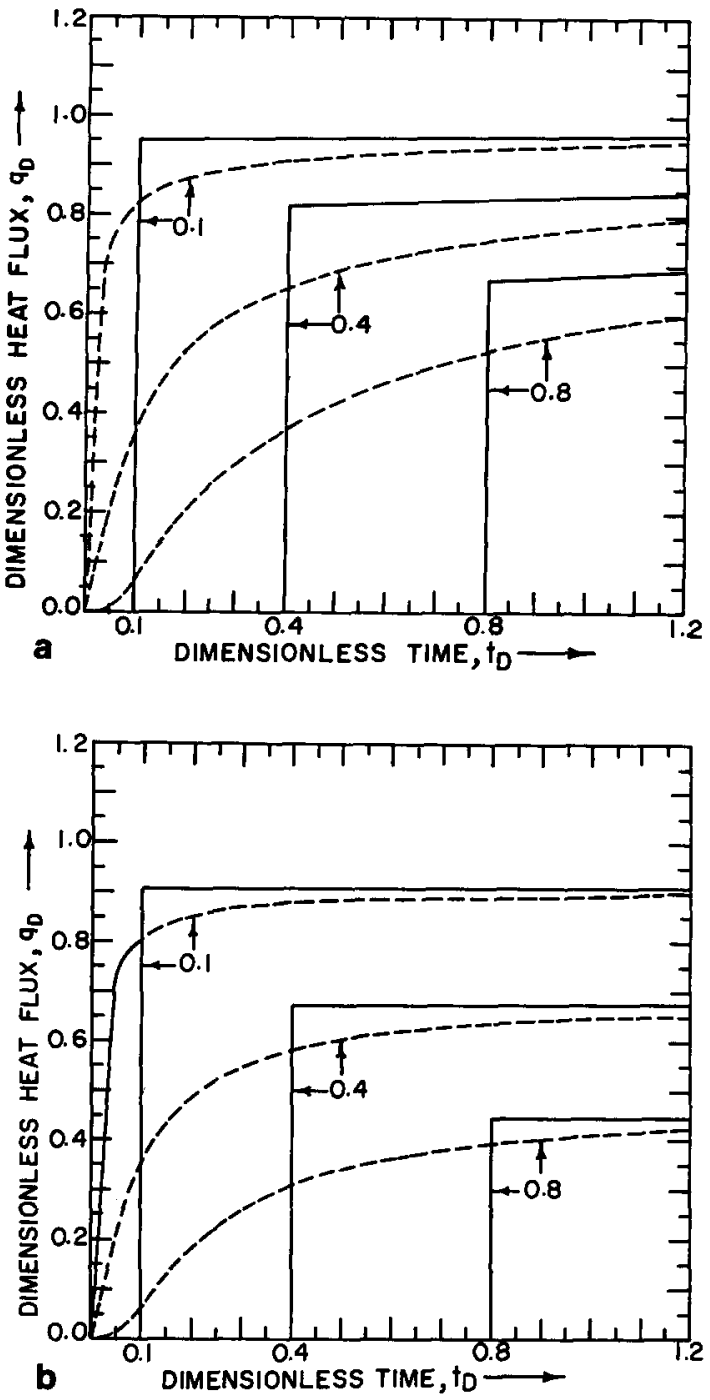

Figure 6 Dimensionless heat flux versus dimensionless time with various wave shape factors: (a) $\phi=0.0$; (b) $\phi=1.0$, for -- , parabolic and - hyperbolic heat conduction equations. Numbers given are dimensionless distance

heat transfer cannot be ignored in the analysis of the wave nature of energy transport, and such heat transfer is prevalent under conditions of larger values of $\tau\left(10^{-3} \mathrm{~s}\right.$ and more $)$, as in the case of materials with a non-homogeneous inner structure or heat conduction at cryogenic temperatures. The results of the present analysis, showing the propagation of desired wave shapes as predicted by hyperbolic heat conduction, are beyond the domain of the classical diffusion theory commonly used in practice.

\section{Acknowledgements}

The author wishes to thank Professor T.N. Subramanian, at the Regional Engineering College, Rourkela, for many valuable suggestions and words of encouragement. The author is also thankful to Professors A.K. Mohanty, R.C. Arora and S. Sarangi of the Indian Institute of Technology, Kharagpur for their in-depth queries during the initiation of this problem. Finally, the help and co-operation of Professor S. Misra, 
Principal of the Regional Engineering College, Rourkela are gratefully acknowledged.

\section{References}

1 Peshkov, V. Second sound in He II J Phys USSR (1944) 8381

2 Morse, M. and Feshbach, H. Methods of Theoretical Physics McGraw-Hill, New York, USA (1953)

3 Vernotte, $P$. Les paradoxes de la theorie continue de l'equation de la chaleur Comptes Rendus Hebd Seances Acad Sci (1958) 246 3154

4 Vernotte, P. La veritable equation de la chaleur Comptes Rendus Hebd Seances Acad Sci (1958) 2472103

5 Cattanee, M.C. Sur une forme de l'equation de la chaleur eliminant le paradoxe d'une propagation instantanee Comptes Rendus Hebd Seances Acad Sci (1958) 247431

6 Maxwell, J.C. On the dynamic theory of gases Phil Trans $R$ Soc (1867) 15749

7 Chester, M. Second sound in solids Physical Rev (1963) 1312013

8 Luikov, A.V. Application of irreversible thermodynamics Int $J$ Heat Mass Transfer (1966) 9139

9 Gembarovic, J. and Majernik, V. Non-Fourier propagation of heat pulses in finite medium Int J Heat Mass Transfer (1988) 31 1073

10 Kaminski, W. Hyperbolic heat conduction equation for materials with a non-homogeneous inner structure Trans ASME J Heat Transfer (1990) 112555

11 Bertman, B. and Sandiford, D.J. Second sound in solid helium Scientific American (1970) 22292

12 Mikic, B.B. A model rate equation for transient thermal conduction Int J Heat Mass Transfer (1967) 101899

13 Baumeister, K.J. and Hamill, T.D. Hyperbolic heat conduction equation-a solution for the semi-infinite body problem Trans ASME J Heat Transfer (1969) 91543

14 Baumeister, K.J. and Hamill, T.D. Hyperbolic heat conduction equation-a solution for the semi-infinite body problem Trans ASME J Heat Transfer (1971) 93126

15 Maurer, M.J. and Thompson, H.A. Non-Fourier effects at high heat flux Trans ASME J Heat Transfer (1973) 95284

16 Wiggert, D.C. Analysis of early-time transient heat conduction by method of characteristics Trans ASME J Heat Transfer (1977) 9935

17 Taitel, Y. On the parabolic, hyperbolic and discrete formulation of heat conduction equation Int $J$ Heat Mass Transfer (1972) 15 369

18 Carey, G.F. and Tsai, M. Hyperbolic heat transfer with reflection Numer Heat Transfer (1982) 5309

19 Vick, B. and Ozisik, M.N. Growth and decay of thermal pulse predicted by the hyperbolic heat conduction equation Trans ASME J Heat Transfer (1983) 105902

20 Ozisik, M.N. and Vick, B. Propagation and reflection of thermal waves in a finite medium Int J Heat Mass Transfer (1984) 271845

21 Frankel, J.I., Vick, B. and Ozisik, M.N. Flux formulation of hyperbolic heat conduction J Appl Phys (1985) 583340

22 Glass, D.E., Ozisik, M.N., McRae, D.S. and Vick, B. On numerical solution of hyperbolic heat conduction Numer Heat Transfer (1985) 8497

23 Glass, D.E., Ozisik, M.N., McRae, D.S. and Vick, B. Hyperbolic heat conduction with temperature-dependent thermal conductivity J Appl Phys (1986) 591861

24 Glass, D.E., Ozisik, M.N. and Vick, B. Hyperbolic heat conduction with surface radiation Int $J$ Heat Mass Transfer (1985) 281823

25 Joseph, D.D. and Prexiosi, L. Heat waves Rev Mod Phys (1989) 6141

26 Ackerman, C.C., Bertman, B., Fairbank, H.A. and Guyer, R.A. Second sound in solid helium Phys Rev Lett (1966) 16789

27 Rogers, S.J. Transport of heat and approach to second sound in some istopically pure alkali-halide crystals Phys Rev (1971) B3 1440

28 Majumdar, A. Microscale heat conduction in dielectric thin films Trans ASME J Heat Transfer (1993) 1157

29 Batemann, H. Tables of Integral Transforms Vol 1, McGrawHill, New York, USA (1954)

30 Carslaw, H.S. and Jaeger, J.C. Conduction of Heat in Solids Oxford University Press, Oxford, UK (1959)

\section{Appendix}

The one-dimensional energy conservation and flux equations for parabolic heat conduction with lateral heat transfer can be obtained from Equations (1) and (5a) in dimensionless form as

$$
\begin{aligned}
& q_{\mathrm{D}}+\frac{\partial T_{D}}{\partial x_{\mathrm{D}}}=0 \\
& \frac{\partial q_{\mathrm{D}}}{\partial x_{\mathrm{D}}}+\frac{\partial T_{\mathrm{D}}}{\partial t_{\mathrm{D}}}+\phi T_{\mathrm{D}}=0
\end{aligned}
$$

These equations can also be obtained from Equations (8a) and $(8 \mathrm{~b})$ by eliminating $\left(\partial q_{\mathrm{D}} / \partial t_{\mathrm{D}}\right)$ in Equation (8b).

The governing differential Equations (A1a) and (A1b), along with its initial conditions given by Equations (9a) and (9b), can be solved by the application of Laplace transformation. The resultant equations in the Laplace plane are

$$
\begin{aligned}
& \frac{\mathrm{d} \bar{q}_{\mathrm{D}}}{\mathrm{d} x_{\mathrm{D}}}=-\bar{T}_{\mathrm{D}}(s+\phi) \\
& \frac{\mathrm{d} \bar{T}_{\mathrm{D}}}{\mathrm{d} x_{\mathrm{D}}}=-\bar{q}_{\mathrm{D}}
\end{aligned}
$$

By eliminating the temperature parameter from the above equations the subsidiary differential equation of dimensionless flux is obtained as

$$
\frac{\mathrm{d}^{2} \bar{q}_{\mathrm{D}}}{\mathrm{d} x_{\mathrm{D}}^{2}}=\bar{q}_{\mathrm{D}}(s+\phi)
$$

Using the boundary conditions given by Equations (12a) and (12b), the solution to the subsidiary differential equation $(\mathrm{A} 3)$ is

$\bar{q}_{\mathrm{D}}=\frac{1}{s} \exp \left[-\sqrt{(s+\phi)} x_{\mathrm{D}}\right]$

From Equations (A2a) and (A4a), the solution for dimensionless temperature in the Laplace plane is

$$
\bar{T}_{\mathrm{D}}=\frac{1}{s \sqrt{(s+\phi)}} \exp \left[-\sqrt{(s+\phi)} x_{\mathrm{D}}\right]
$$

Using the table of Laplace inversion given in work by Carslaw and Jaeger ${ }^{3}$, the solutions of Equations $(\mathrm{A} 4 \mathrm{a})$ and $(\mathrm{A} 4 \mathrm{~b})$ are

$$
\begin{aligned}
q_{\mathrm{D}}=\frac{1}{2}\{ & \left.\exp \left[-x_{\mathrm{D}} \sqrt{(\phi)}\right] \operatorname{erfc}\left[x_{\mathrm{D}} /\left(2 \sqrt{t_{\mathrm{D}}}\right)-\sqrt{\left(\phi t_{\mathrm{D}}\right.}\right)\right] \\
& \left.\left.+\exp \left[x_{\mathrm{D}} \sqrt{(\phi)}\right] \operatorname{erfc}\left[x_{\mathrm{D}} /\left(2 \sqrt{t_{\mathrm{D}}}\right)+\sqrt{\left(\phi t_{\mathrm{D}}\right.}\right)\right]\right\}
\end{aligned}
$$

$$
\begin{aligned}
T_{\mathrm{D}}= & {[1 / 2 \sqrt{(\phi)}]\left\{\operatorname { e x p } [ - x _ { \mathrm { D } } \sqrt { ( \phi ) } ] \operatorname { e r f c } \left[x_{\mathrm{D}} /\left(2 \sqrt{t_{\mathrm{D}}}\right)\right.\right.} \\
\left.-\sqrt{\left(\phi t_{\mathrm{D}}\right)}\right]-\exp \left[x_{\mathrm{D}} \sqrt{(\phi)}\right] & \operatorname{erfc}\left[x_{\mathrm{D}} /\left(2 \sqrt{t_{\mathrm{D}}}\right)\right. \\
& \left.\left.\left.+\sqrt{\left(\phi t_{\mathrm{D}}\right.}\right)\right]\right\}
\end{aligned}
$$

Under limiting values of the dimensionless parameters, Equations (A5a) and (A5b) can be modified as follows. 


\section{Case 1: $\phi \neq 0, \mathrm{x}_{D} \rightarrow 0$}

The surface temperature is obtained from Equation (A5b) as

$$
T_{\mathrm{D}}=\operatorname{erf}\left[\sqrt{\left(\phi t_{\mathrm{D}}\right)}\right] / \sqrt{\phi}
$$

\section{Case 2: $\phi \rightarrow 0, \mathrm{x}_{D} \neq 0$}

Without lateral heat transfer, Equation (A5b) yields an indeterminate form. But its solution can be obtained by Laplace inversion of Equation (A4b) with $\phi \rightarrow 0$ from the inversion table ${ }^{30}$ as

$$
\begin{aligned}
T_{\mathrm{D}}=2\left\{\sqrt{\left(t_{\mathrm{D}} / \pi\right)} \exp \left(-x_{\mathrm{D}}^{2} / 4 t_{\mathrm{D}}\right)\right. \\
\left.-\left(x_{\mathrm{D}} / 2\right) \operatorname{erfc}\left[x_{\mathrm{D}} /\left(2 \sqrt{t_{\mathrm{D}}}\right)\right]\right\}
\end{aligned}
$$

Case 3: $\phi \rightarrow 0, x_{D} \rightarrow 0$

The surface temperature from the previous case can be obtained by substituting $x_{\mathrm{D}}=0$ in Equation (A6b). The resultant equation is

$$
T_{\mathrm{D}}=2 \sqrt{\left(t_{\mathrm{D}} / \pi\right)}
$$

